\title{
Retracted: Computational study of hydrogen adsorption on potassium-decorated boron nitride nanotubes
}

\author{
Md Shahzad Khan and Mohd Shahid Khan ${ }^{*}$
}

\begin{abstract}
Retraction
This article was mistakenly published twice. For this reason this duplicate article has now been retracted. For citation purposes please cite the original: http://www.inljournal.com/?_action=articlelnfo\&article=19

\section{Abstract}

We have investigated the potassium-decorated boron nitride nanotubes for hydrogen storage using semi-empirical AM1 method. The ultra narrow $(3,3)$ and $(5,0)$ boron nitride nanotubes of same diameter but of different chirality have been used. Both of them show hydrogen storage greater than $8 \%$ by weight. Density of states have been calculated, and it is found that the presence of alpha density of state of potassium results in smaller energy gap; as a result of which, the conductivity of the potassium-decorated boron nitride nanotubes is enhanced as compared to pristine boron nitride nanotubes. Charge decomposition analysis showed that there is significant transfer of charge from adsorbate potassium to boron nitride nanotubes; the same is also confirmed by Mulliken population analysis. For same diameter, due to different electronic configuration, zigzag tube is found to be slightly more favorable for hydrogen adsorption. The results of the present simulation study suggest that the potassiumdecorated boron nitride nanotubes are good candidate for hydrogen adsorption.
\end{abstract}

Keywords: Potassium decorated, Boron nitride nanotubes, Semi-empirical method, Hydrogen storage, Binding energy, Density of states

\section{Background}

Safe and efficient hydrogen adsorption requirement for future fuel cell vehicle has raised the demand for simulation study of adsorption of hydrogen on nanostructures. Until recently, several studies have been carried out on carbonbased materials such as nanotubes [1-6], fullerenes [7,8], metal hydride, and metal-organic frame works $[9,10]$. The binding energy of adsorption of hydrogen on the pristine single-wall carbon nanotube (SWCNT) is very small [5]. However, it has been reported that the carbon nanomaterials when coated with alkali and alkaline earth atoms can be used for hydrogen storage [11-13]. In contrast to SWCNTs which are semiconductors or metallics, depending on their diameter and helicities, the boron nitride nanotubes (BNNTs) having features similar to carbon nanotubes are wide-band-gap semiconductors with their

\footnotetext{
* Correspondence: mskhan@jmi.ac.in; shahidkhan_m@yahoo.com

Department of Physics, Jamia Millia Islamia (Central University), New Delhi, 110025, India
}

band gap just independent of tube diameter and helicity [14]. Also, boron nitride nanotubes are thermally and chemically more stable [15-17]. These unique properties of BNNTs open a path for interesting research in order to examine these as potential candidate for hydrogen adsorption. It has been reported by Ma et al. [18] that the multiwalled and bamboo-like BNNTs can absorb hydrogen up to $2.6 \mathrm{wt} . \%$ at room temperature wherein $70 \%$ of the hydrogen is chemisorbed. Tang et al. [19] found that hydrogen storage can even reach up to $4.2 \mathrm{wt} . \%$ if the BNNTs were treated with platinum. These theoretical studies suggested that metal-doped BNNTs hold some potential as hydrogen storage media. The Pt-doped [20], Ti-doped [21], and Si-doped [22] BNNTs have been examined, and the results of these studies proved that the capacity for hydrogen storage in the doped BNNTs is enhanced as a result of doping (or decorating) as compared to pristine BNNTs and BN clusters. These studies have also highlighted the importance of the 
(a)
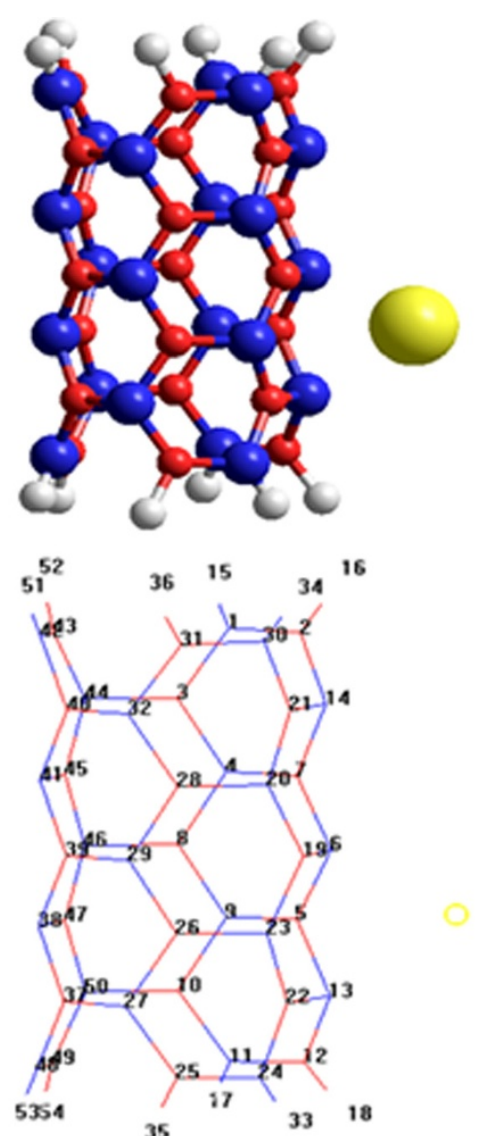

(b)
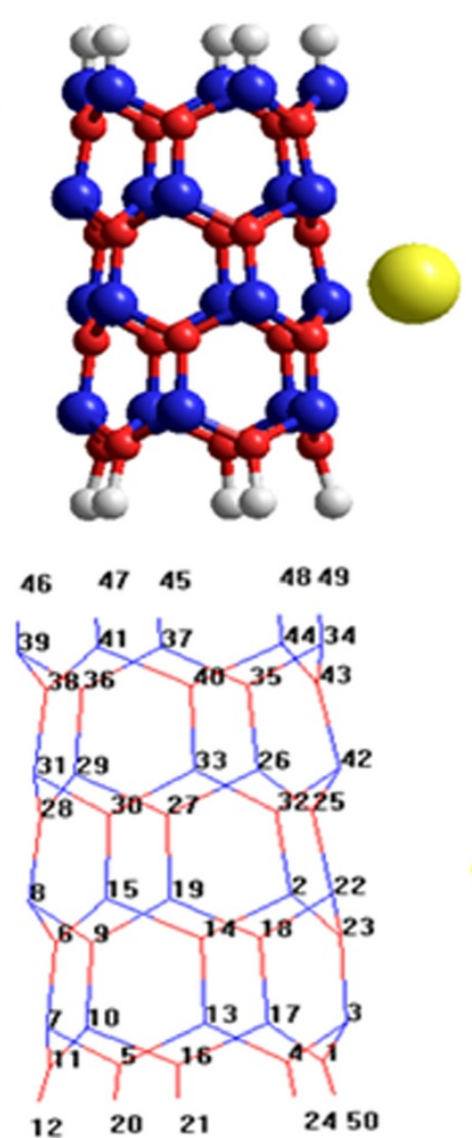

Figure 1 AM1-optimized structure of K(yellow)-decorated (a) $(3,3)$ and (b) $(5,0)$ BNNTs. Red ball for nitrogen and blue for boron atom.

surface area of BNNTs in hydrogen uptake of the nanostructures [23].

Keeping these findings in mind, we have selected relatively light alkali earth metal as a candidate for decoration on hydrogen-terminated BNNTs. Hydrogen termination has been used to avoid the boundary effect. Potassium, being a light metal, gives little weight contribution to effective storage material; however, adsorbing good amount of hydrogen molecules leads to increase of the hydrogen storage by weight. The aim of the present work is to examine the extent up to which the K-decorated BNNTs enhance the hydrogen uptake using the semi-empirical AM1 method [24], which has been used widely to calculate various properties at small computational cost for wide variety of materials [25-32].

\section{Methods}

Model system and computational method

The geometry optimizations of all the structures leading to energy minima were carried using the AM1 self-consistent field molecular orbital method at the unrestricted Hartree Fock level. The structures were initially optimized using the

Table 1 Changes of bond length of the associated mesh of BNNTs after doping and adsorption of potassium

\begin{tabular}{|c|c|c|c|c|c|}
\hline \multicolumn{3}{|c|}{$(3,3)$ BNNT } & \multicolumn{3}{|c|}{$(5,0)$ BNNT } \\
\hline Bond lengthin (Å) & PristineBNNT & K-dopedBNNT & Bond lengthin $(\AA)$ & PristineBNNT & K-dopedBNNT \\
\hline B5-B13 & 1.4653 & 1.5064 & B25-N42 & 1.4655 & 1.5045 \\
\hline B5-N6 & 1.4601 & 1.4435 & $\mathrm{~B} 25-\mathrm{N} 22$ & 1.4437 & 1.4321 \\
\hline B19-N6 & 1.4596 & 1.5927 & B23-N22 & 1.4675 & 1.5398 \\
\hline B19-N23 & 1.4523 & 1.5100 & B23-N2 & 1.4675 & 1.5398 \\
\hline B22-N13 & 1.4432 & 1.4831 & B32-N42 & 1.4655 & 1.5045 \\
\hline B22-N23 & 1.4690 & 1.4510 & B32-N2 & 1.4675 & 1.4321 \\
\hline
\end{tabular}


$\mathrm{MM}+$ molecular mechanics method and then were optimized using AM1 method employing the steepest-descent optimizer followed by conjugate gradient, Fletcher-Reeves and Polak-Ribiere methods consecutively with convergence limit of $0.0001 \mathrm{kcal} / \mathrm{mol}$, and RMS gradient limit of $0.001 \mathrm{kcal} / \mathrm{A}-\mathrm{mol}$. All the calculations were performed using the HyperChem (7.1) package program [33]. For the charge decomposition analysis and phonon density of states (PDOS), the single point calculations were carried out and the results of which were then used as input for the AOMIX program $[34,35]$.

\section{Results and discussion}

Armchair $(3,3)$ and zigzag $(5,0)$ ultra-narrow boron nitride nanotubes terminated with hydrogen atoms, each having 15 meshes, have been selected for the present study. Both of these have different chirality but are diametrically almost
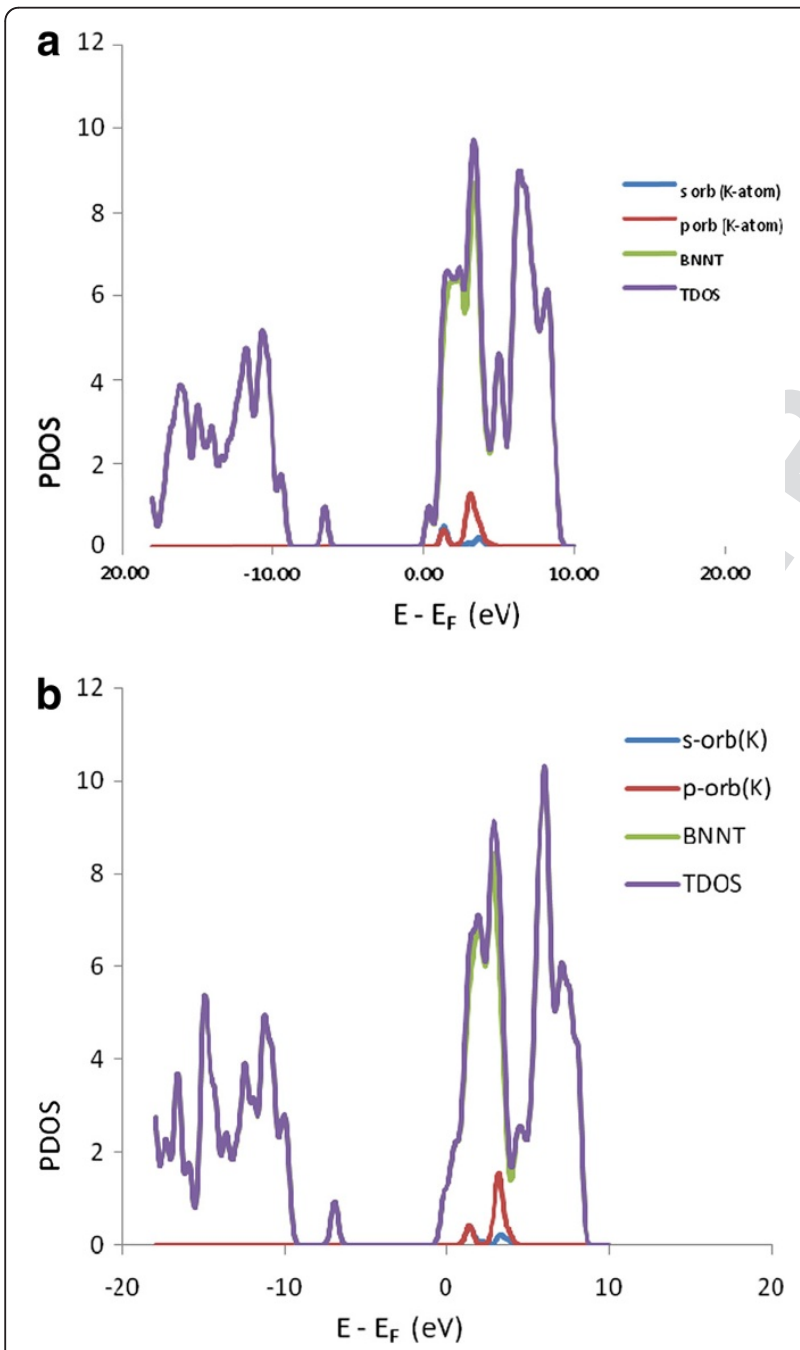

Figure 2 PDOS for K-doped $(3,3)$ (a) and $(5,0)$ (b) BNNTs are plotted. Color on line represents the various fragments. equal and both of these are wide-band-gap semiconductors. Figure 1a,b shows the AM1-optimized structures obtained after one $\mathrm{K}$ atom was relaxed in the neighborhood of the already optimized structure of the pristine $(3,3)$ and $(5,0)$ BNNTs, respectively. As can be seen from these figures, AM1 force-field optimization resulted in the adsorption of the $\mathrm{K}$ atom on the surface of the exterior wall of the optimized structures of $(3,3)$ and $(5,0)$ BNNTs. The AM1-optimized bond lengths in the mesh near the dopant $\mathrm{K}$ atom before and after doping potassium atom on the $(3,3)$ and $(5,0)$ BNNTs are listed in Table 1 from which a distortion of BNNTs after potassium doping can be observed. The same is also apparent in Figure 1a,b from which it can be seen that the K atom occupies the hollow site on one of the hexagons of the BNNT. Adsorption energy of $\mathrm{K}$ atom doped on BNNT is evaluated according to the expression:

$(3,3)$

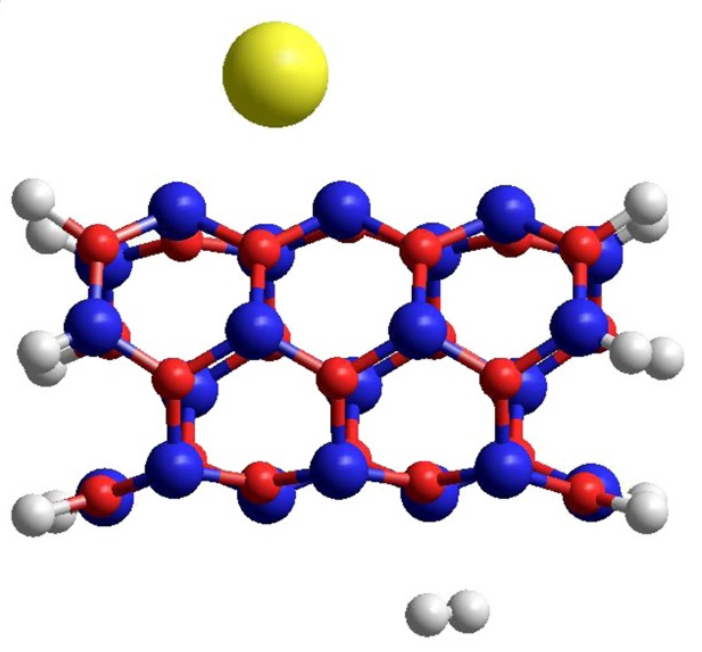

$(5,0)$

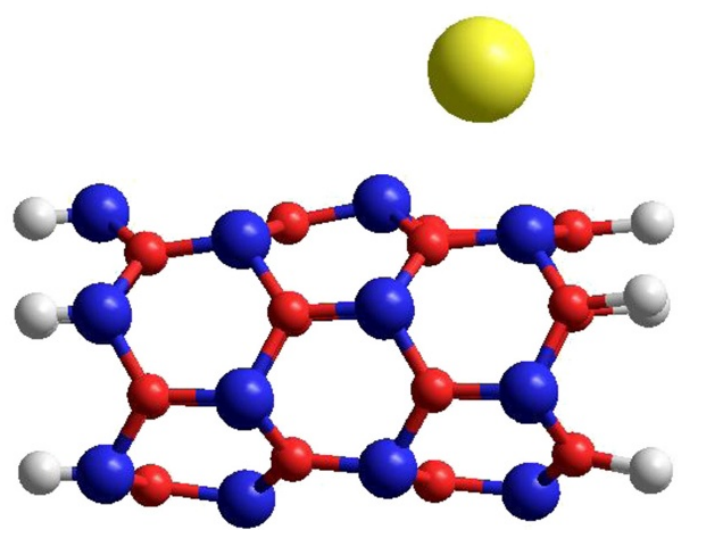

Figure $3 \mathrm{H}_{2}$ molecule adsorbed on potassium-decorated $(3,3)$ and $(5,0)$ BNNTs. 
(a)

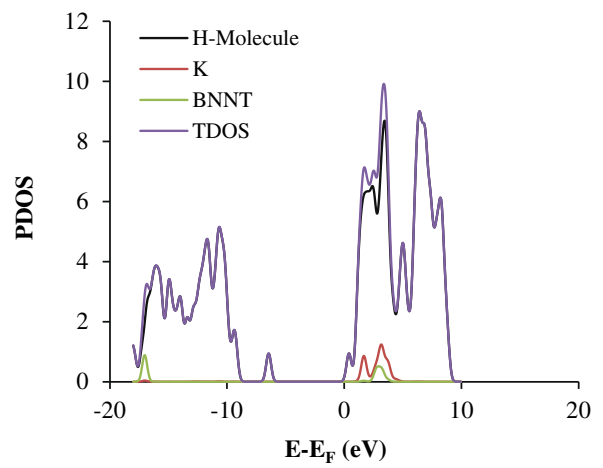

(b)

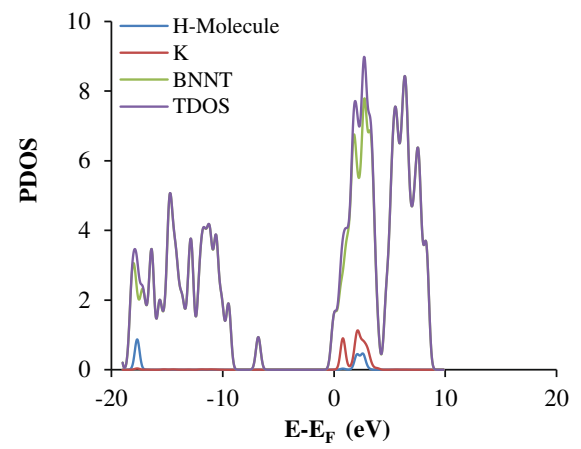

Figure 4 PDOS for single $\mathbf{H}_{\mathbf{2}+}$ K-decorated (3,3) BNNT. Color online are fragment-wise selection, (a) for $(3,3)$ and $(\mathbf{b})$ for $(5,0)$.

$$
E_{\mathrm{ads}}=E(\mathrm{NT}+\mathrm{K})-E(\mathrm{NT})-E(\mathrm{~K})
$$

where $E(\mathrm{NT}+\mathrm{K})$ is energy of $\mathrm{K}$-doped $\mathrm{BNNT}, E(\mathrm{NT})$ is energy of nanotube, and $E(\mathrm{~K})$ is free energy of potassium atom.

The process of adsorbing $\mathrm{K}$ atom on BNNTs is found to be exothermic with binding energy of 3.2 and $2.8 \mathrm{eV} / \mathrm{K}$ atom for $(3,3)$ and $(5,0)$ BNNTs, respectively. Margine and Crespi [36] reported that the presence of strong electron donor alkali metals on BNNTs provided positive potential surrounding alkali atoms. The calculated dipole moment of 9.5 D and 9.7 D for $(3,3)$ and $(5,0)$ K-doped BNNTs, respectively, is relatively large because of the presence of potassium atom that is donating the positive charge to the BNNT. The large enhancement in the calculated value of the dipole moment of the BNNT has also been predicted using advance level of theory by Farmanzabeh and Ghazanfary [37]. The same is also supported by Mulliken charge analysis which revealed a charge transfer of $0.697 e^{-}$ from decorated potassium atom to $(3,3) \mathrm{BNNT}$ and $0.71 e^{-}$ to $(5,0)$ BNNT, respectively. The redistribution of charge within BNNT is markedly over the mesh which is nearest to potassium atom. The boron constituent still has positive charge which has been slightly reduced, while negative charge on nitrogen has increased a little. The main contributions to the highest occupied molecular orbital (HOMO) and lowest unoccupied molecular orbital (LUMO) in Kdoped BNNTs are from nearest neighboring atoms of potassium and have feeble participation of $s$ and $p$ orbitals from potassium side; on the other hand, for pristine BNNTs, the HOMO is largely consisted of $p$ orbital of constituent nitrogen atoms, and LUMO consists of $s$ orbital of boron atoms. In order to examine the effect of $\mathrm{K}$ doping on the electronic structure of BNNTs, the PDOS of the doped BNNTs are plotted and are shown in Figure 2. It is the presence of potassium atom due to which the contribution to inter-frontier orbitals by the labeled constituents got manifested. It is the alpha DOS of decorated sample that has the change in electronic state. The conductivity of BNNTs gets enhanced due to the appearance of a peak on the top of the valence band in the presence of potassium adsorbate.

\section{Adsorption of a single $\mathrm{H}_{2}$}

The $\mathrm{H}_{2}$ molecule can be adsorbed to the K-decorated BNNTs through $\mathrm{K}$ atom with distance of 2.5 and $2.49 \AA$ from $\mathrm{K}$ atom in $(3,3)$ and $(5,0) \mathrm{K}$-decorated BNNTs, respectively. The two $\mathrm{H}-\mathrm{K}$ bond lengths are 2.52 and 2.54 $\AA$ for both the $(3,3)$ and $(5,0)$ BNNTs; however, there is slight elongation in $\mathrm{H}-\mathrm{H}$ bond length in $(5,0)$ BNNT. Binding-energy value is $0.53 \mathrm{eV} / \mathrm{H}_{2}$ molecule for K-doped $(3,3)$ BNNT while in the case of $(5,0)$ nanotube it is slightly less, $0.51 \mathrm{eV} / \mathrm{H}_{2}$ molecule. The predicted adsorption energy is defined as:

$$
E_{\mathrm{ads}}=E\left(\mathrm{~K}+\mathrm{BNNT}+n \mathrm{H}_{2}\right)-E\left[\mathrm{~K}+\mathrm{BNNT}-(n-1) \mathrm{H}_{2}\right]-E\left(\mathrm{H}_{2}\right)
$$

where $n$ is the number of free hydrogen molecule.

The orientation of hydrogen molecule is side-on with respect to $\mathrm{K}$ atom in both the nanotubes as can be seen in Figure 3. Figure 4 shows the PDOS of the single $\mathrm{H}_{2}$ molecule adsorbed on the $(3,3)$ and $(5,0) \mathrm{K}$-decorated BNNTs. The PDOS analysis, by considering $\mathrm{H}_{2}$ molecule,

Table 2 Binding energy per hydrogen molecule (in eV) on potassium-decorated BNNT

\begin{tabular}{lllllllll}
\hline & $\mathbf{1 H}_{\mathbf{2}}$ & $\mathbf{2} \mathbf{H}_{\mathbf{2}}$ & $\mathbf{3} \mathbf{H}_{\mathbf{2}}$ & $\mathbf{4 \mathbf { H } _ { \mathbf { 2 } }}$ & $\mathbf{5 \mathbf { H } _ { \mathbf { 2 } }}$ & $\mathbf{6} \mathbf{H}_{\mathbf{2}}$ & $\mathbf{7 \mathbf { H } _ { \mathbf { 2 } }}$ & $\mathbf{8} \mathbf{H}_{\mathbf{2}}$ \\
\hline$(3,3) \mathrm{K}$-doped BNNT & 0.51 & 0.49 & 0.48 & 0.45 & 0.43 & 0.41 & 0.40 & 0.38 \\
$(5,0) \mathrm{K}$-doped BNNT & 0.53 & 0.51 & 0.50 & 0.48 & 0.46 & 0.44 & 0.42 & 0.41 \\
\hline
\end{tabular}



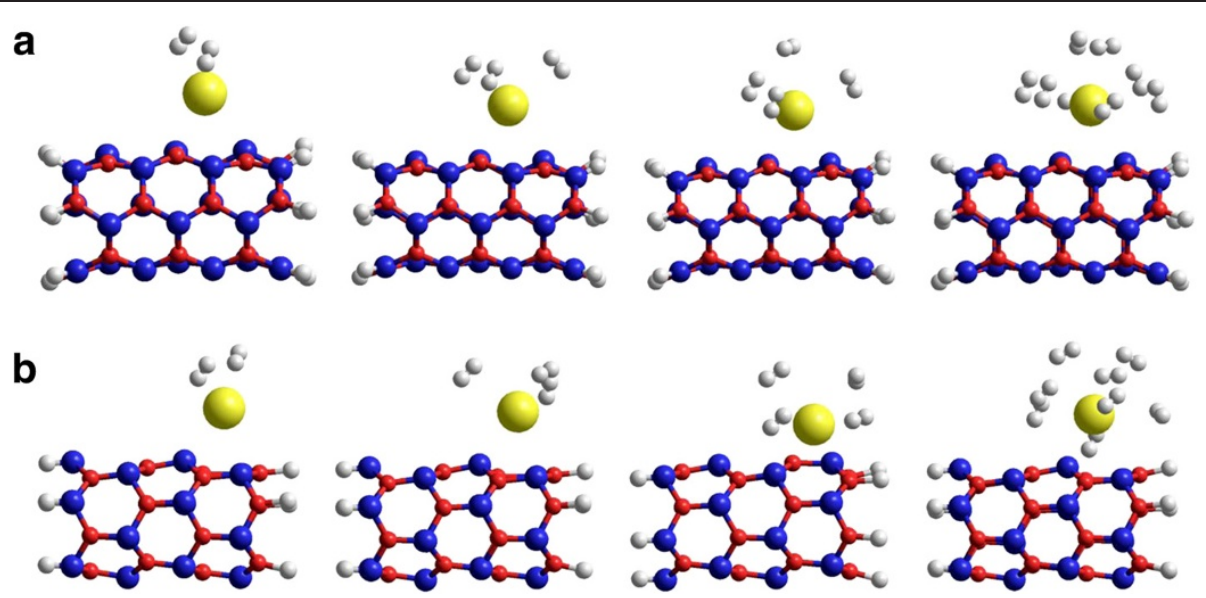

Figure 5 Multiple hydrogen adsorption on K-decorated (a) $(3,3)$ and (b) $(5,0)$ BNNTs.

potassium atom, and BNNT as individual fragments, revealed that it is the hybridization of the empty $\mathrm{K}-4 p$ orbitals with $\mathrm{H}_{2} \sigma$ orbital that contributes to the $\mathrm{H}_{2}$ adsorption for both $(3,3)$ and $(5,0)$ nanotubes. The charge decomposition analysis considering $\mathrm{K}$ atom and a single $\mathrm{H}_{2}$ molecule as fragment- 1 and $(3,3)$ BNNT as fragment-2 revealed that there is a net charge donation of $0.847 e^{-}$for alpha MOs from fragment-1 to fragment- 2 in case of alpha DOS; for beta DOS, there is a charge donation of $0.156 e^{-}$from fragment- 2 to fragment- 1 . Similarly, for Kdoped $(5,0)$ BNNT, a net charge donation of $0.853 e^{-}$from fragment- 1 to fragment- 2 takes place for alpha DOS, whereas a net charge donation of $0.148 e^{-}$is predicted from fragment-2 to fragment-1 in case of beta MOs. These donations are net result of donation from fragment- 1 to fragment- 2 and back-donation from fragment- 2 to fragment-1. The self-polarization of fragment orbital plays almost negligible role in charge transfer. The Mullikan population analysis revealed that the hydrogen molecule has unequal charges (viz $0.050 e^{-}$and $0.029 e^{-}$) on its two atomic centers, and it is slightly polar in nature as a result of which it seeks $\mathrm{K}$ atom which carry $0.612 e^{-}$ charge over it. It is suggested that the charge transfer leaves the metal dopant in cationic form so that the hydrogen molecule can be attracted by the metal cation via the charge polarization mechanism.

\section{Adsorption of multiple $\mathrm{H}_{2}$}

The adsorption of the second $\mathrm{H}_{2}$ molecule resulted in a decrease in binding energy per $\mathrm{H}_{2}$ molecule for both $(3,3)$ and $(5,0) \mathrm{K}$-doped BNNTs with values 0.49 and $0.51 \mathrm{eV} /$ $\mathrm{H}_{2}$, respectively. There is almost no change in bond length of $\mathrm{H}_{2}$ molecules. Mulliken population analysis has shown that it is only the redistribution of charges between $\mathrm{K}$ atom and $\mathrm{H}_{2}$ molecules which is responsible for binding of the second $\mathrm{H}_{2}$ molecule. The charge configuration on the surface of BNNTs is affected negligibly by adsorption of the second hydrogen molecules. The $\mathrm{K}$ atom is found to have a slightly reduced charge of $0.544 e^{-}$. On the other hand, the hydrogen molecules are observed to have

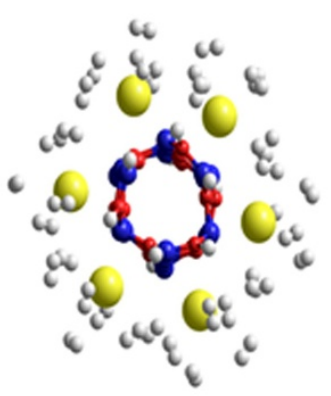

$8.5 \%$ by wt of Hydrogen on $(3,3)$ BNNT

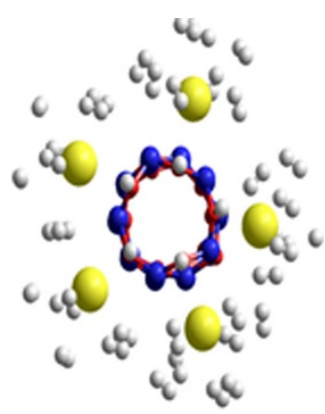

$9.4 \%$ by wt of Hydrogen on $(5,0)$ BNNT

Figure 6 Coverage of $\mathrm{K}$ atom on $(\mathbf{3}, \mathbf{3})$ and $(\mathbf{5}, \mathbf{0})$ nanotubes. Two-fifth coverage of $\mathrm{K}$ atom on $(3,3)$ nanotube with $36 \mathrm{H}_{2}$ molecules adsorbed and one third coverage on $(5,0)$ nanotube with $37 \mathrm{H}_{2}$ molecules. 
unequal distribution of charge within its hydrogen atoms with values of $0.058 e^{-}$and $0.018 e^{-}$for the first $\mathrm{H}_{2}$ and $0.059 e^{-}$and $0.014 e^{-}$on the second $\mathrm{H}_{2}$. On progressively adding more hydrogen molecules, the charge on $\mathrm{K}$ atom is seen to decrease. The charge on $\mathrm{K}$ atom is found to be $0.339 e^{-}$for the adsorption of eight $\mathrm{H}_{2}$ molecules. The optimization calculations for different numbers of $\mathrm{H}_{2}$ molecules for the adsorption on K-doped BNNTs were carried out, and the calculated values of binding energy per hydrogen are presented in Table 2. It is found that one $\mathrm{K}$ atom can at most adsorb eight $\mathrm{H}_{2}$ molecules with $E_{\mathrm{b}}$ of 0.38 and $0.41 \mathrm{eV} / \mathrm{H}_{2}$ for $(3,3)$ and $(5,0)$ BNNTs, respectively. Multiple hydrogen molecules adsorbed on K-doped BNNTs are shown in Figure 5. These results indicate that the binding energy of $\mathrm{H}_{2}$ has little to do with the chirality of BNNTs.

\section{Hydrogen adsorption on high-coverage K-decorated BNNT}

A further study for the stable $\mathrm{K}$ adsorbates with high coverage on boron nitride nanotube resulted in a maximum coverage of two fifth on the $(3,3)$ and one third on $(5,0)$ nanotube with $E_{\mathrm{b}}$ of 2.3 and $2.8 \mathrm{eV}$, respectively. For $(5,0) \mathrm{BNNT}$, a maximum coverage of one third with $\mathrm{K}$ atoms can be made as clustering of $\mathrm{K}$ atoms started with addition of more potassium atoms due to the nearest neighboring effect; as for the stable $\mathrm{K}$ adsorbates on boron nitride nanotube, K-K distances should be larger than that of bulk K. Similarly, maximum K converge of two fifth is possible on $(3,3)$ BNNT with $6-\mathrm{K}$ atoms. The $(3,3) \mathrm{K}$ decorated BNNT with maximum coverage of two fifth is found to adsorb at most $36 \mathrm{H}_{2}$ with $E_{\mathrm{b}}$ of $0.41 \mathrm{eV} / \mathrm{H}_{2}$, corresponding to a hydrogen uptake of $8.5 \%$ by weight. For $(5,0) \mathrm{K}$-doped BNNT with maximum coverage, the number of $\mathrm{H}_{2}$ molecules adsorbed is found to be 37 with $E_{\mathrm{b}}$ of $0.55 \mathrm{eV} / \mathrm{H}_{2}$ with uptake of $9.4 \%$ by weight. The optimized molecular structures of these K-decorated BNNTs with maximum coverage and possible adsorbed hydrogen molecules are shown in Figure 6. It can be inferred from these results that the zigzag $(5,0) \mathrm{K}$-decorated BNNTs are more favorable for hydrogen adsorption.

\section{Conclusions}

The optimizations based on semi-empirical AM1 method have been carried out for exploring the adsorption of the hydrogen on K-decorated BNNTs with chirality $(3,3)$ and $(5,0)$. It is found from the calculated PDOS for Kdecorated BNNTs that the conductivity of these K-decorated BNNTs is increased as a result of decoration with $\mathrm{K}$ atoms due to the change in electron density of state of pristine nanotube. Charge decomposition analysis has indicated transfer of a significant amount of charge to nanotube from adsorbed $\mathrm{K}$ atom and $\mathrm{H}_{2}$ molecule. It is the hybridization of empty K-4 $p$ orbital with $\mathrm{H}_{2} \sigma$ orbital that leads to hydrogen adsorption. The binding energy of the $\mathrm{K}$ atom is found to be independent of the chirality of the BNNTs. The coverage of K over nanotubes should not be much larger as it causes clustering over nanotube. The maximum coverage of $\mathrm{K}$ atoms on $(3,3)$ and $(5,0)$ BNNTs resulted in $8.5 \%$ and $9.4 \%$ hydrogen storage by weight on BNNTs, respectively. These results of the present simulation study suggest that the K-decorated BNNTs are good candidates for hydrogen adsorption.

\section{Competing interests}

The authors declare that they have no competing interests.

\section{Authors' contributions}

MSK carried out the MO calculations, was involved in the design of the study, analysis of the results and prepared the draft of the manuscript. MSK* conceived of the study, participated in its design and analysis, and revised the manuscript critically to give it final shape for publication. All authors read and approved the final manuscript.

\section{Authors' information}

Md. Shahzad Khan obtained his M.Sc. Physics degree from Jamia Millia Islamia, New Delhi in 2008 and is currently pursuing Ph.D. in Physics from Jamia Millia Islamia, New Delhi.

Mohd. Shahid Khan did his M.Sc. Physics (Materials Science) from Jamia Millia Islamia, New Delhi in 1992. He completed his doctoral thesis for Ph.D. Physics from Jamia Millia Islamia in 2002. Dr. Khan joined Department of Physics as Assistant Professor in 2006. His areas of research are Laser spectroscopy, non-linear optics, and Computational Materials Science \& Nanoscience.

\section{Acknowledgments}

One of the authors, MSK, acknowledges the grant of financial support from Department of Science and Technology, New Delhi under the International Travel Support Scheme for attending and presenting this work in the XIX IMRC at Cancun, Mexico from August15 to 19, 2010.

Received: 22 January 2011 Published: 27 April 2012

\section{References}

1. Dillon, A.C., Jones, K.M., Bekkendahl, T.A., Kiang, C.H., Bethune, D.S.: Heben. MJ:Storage of hydrogen in single-walled carbon nanotubes. Nature. $\mathbf{3 8 6}$ 377-379 (1997)

2. Chan, S.P., Chen, G., Gong, X.G., Liu, Z.F.: Chemisorption of Hydrogen Molecules on Carbon Nanotubes under High Pressure. Phys. Rev. Lett. 87, 205502-205505 (2001)

3. Gulseren, O., Yildirim, T., Ciraci, S.: Tunable Adsorption on Carbon Nanotubes. Phys. Rev. Lett. 87, 116802-116805 (2001)

4. Deng, W.-Q., Goddard, Xu-X, Wa III: Enhancement of hydrogen physisorption on graphene and carbon nanotubes by Li doping. Phys. Rev. Lett. 9(2), 166103-116108 (2004)

5. Dag, S., Ozturk, Y., Ciraci, S., Yildirim, T.: Adsorption and dissociation of hydrogen molecules on bare and functionalized carbon nanotubes. Phys. Rev. B. 72, 155404-155411 (2005)

6. Yildirim, T., Ciraci, S.: Titanium-Decorated Carbon Nanotubes as a Potential High-Capacity Hydrogen Storage Medium. Phys. Rev. Lett. 94, 175501-175504 (2005)

7. Zhao, Y., Kim, Y.-H., Dillon, A.C., Heben, M.J., Zhang, B.: Hydrogen Storage in Novel Organometallic Buckyballs. Phys. Rev. Lett. 94, 155504-155507 (2005)

8. Yildirim, T., Iniguez, J., Ciraci, S.: Molecular and dissociative adsorption of multiple hydrogen molecules on transition metal decorated $C_{60}$. Phys. Rev. B. 72, 153403-153406 (2005)

9. Bogdanovic, B., Felderhoff, M., Kaskel, S., Pommerin, A., Schlichte, K., Schuth, F.: Improved hydrogen storage [properties of Ti-doped sodium analate using Titanim nanoparticles as doping agents. Adv. Mater. 15, 1012-1015 (2003)

10. Yildirim, T., Hartman, M.R.: Direct Observation of Hydrogen Adsorption Sites and Nanocage Formation in Metal-Organic Frameworks. Phys. Rev. Lett. 95 215504-215507 (2005) 
11. Yoon, M., Yang, S., Hicke, C., Wang, E., Geohegan, D., Zhang, Z.: Calcium as the Superior Coating Metal in Functionalization of Carbon Fullerenes for High-Capacity Hydrogen Storage. Phys. Rev. Lett. 100, 206806-206809 (2008)

12. Lee, H., Ihm, J., Cohen, M.L., Louie, S.G.: Calcium-decorated carbon nanotubes for high-capacity hydrogen storage: First-principles calculations. Phys. Rev. B. 80, 115412-115416 (2009)

13. Liu, W., Zhao, Y.H., Li, Y., Jiang, Q., Lavernia, E.J.: Enhanced Hydrogen Storage on Li-Dispersed Carbon Nanotubes. J. Phys. Chem. C. 113, 2028-2033 (2009)

14. Blase, X., Rubio, A., Louie, S.G., Cohen, M.L.: Stability and Band Gap Constancy of Boron Nitride Nanotubes. Europhys. Lett. 28, 335-340 (1994)

15. Loiseau, A., Willaime, F., Demoncy, N., Hug, G., Pascard, H.: Boron Nitride Nanotubes with Reduced Numbers of Layers Synthesized by Arc Discharge. Phys. Rev. Lett. 76, 4737-4740 (1996)

16. Bengu, E., Marks, L.D.: Single-Walled BN Nanostructures. Phys. Rev. Lett. 86 2385-2387 (2001)

17. Nirmala, V., Kolandaivel, P.: Structure and electronic properties of armchair boron nitride nanotubes. J. Mol. Struct. (Theochem) 817, 137-145 (2007)

18. Ma, R., Bando, Y., Zhu, H., Sato, T., Xu, C., Wu, D.H.: Hydrogen Uptake in Boron Nitride Nanotubes at Room Temperature. J. Am. Chem. Soc. 124, 7672-7673 (2002)

19. Tang, C., Bando, Y., Ding, X.X., Qi, S., Golberg, D.: Catalyzed Collapse and Enhanced Hydrogen Storage of BN Nanotubes. J. Am. Chem. Soc. 124 14550-14551 (2002)

20. Wu, X., Yang, J.L., Zeng, X.C.: Adsorption of hydrogen molecules on the platinum-doped boron nitride nanotube. J. Chem. Phys. 125, 044704-044709 (2006)

21. Durgun, E., Jang, Y.R., Ciraci, S.: Hydrogen storage capacity of Ti-doped boron-nitride and BBe-substituted carbon nanotubes. Phys. Rev. B. 76 073413-073416 (2007)

22. Shevlin, S.A., Guo, Z.X.: Hydrogen sorption in defective hexagonal BN sheets and BN nanotubes. Phys. Rev. B. 76, 024104-024113 (2007)

23. Seayad, A.M., Antonelli, D.M.: Recent Advances in Hydrogen Storage in Metal-Containing Inorganic Nanostructures and Related Materials. Adv. Mater. 16, 765-777 (2004)

24. Dewar, M.J.S., Zoebisch, E.G., Healy, E.F., Stewart, J.J.P.: Development and use of quantum mechanical molecular models. 76. AM1: a new general purpose quantum mechanical molecular model. J. Am. Chem. Soc. 107, 3902-3909 (1985)

25. Erkoc, S.: Structural and electronic properties of single-wall BN nanotubes. J. Mol. Struct. (Theochem). 542, 89-93 (2001)

26. Turker, L.: Certain endohedrally hydrogen doped Be@C60 systems theoretical study. J. Mol. Struct. (Theochem) 577, 205-211 (2002)

27. Khan, M.S., Khan, Z.H.: Electronic Absorption Spectra of Hydroxy-Substituted Anthraquinones and Their Interpretation using ZINDO/S and AM1 Methods. Cand. J. Anal. Sci. Spectrosc. 47, 146-156 (2002)

28. Khan, M.S., Khan, Z.H.: Electronic absorption spectra of amino substituted anthraquinones and their interpretation using ZINDO/S and AM1 methods. Spectrochim. Acta. A. 59, 1409-1426 (2003)

29. Turker, L.: AM 1 treatment of endohedrally hydrogen doped C56 systems, nH2@C56. J. Mol. Struct. (Theochem). J. Mol. Struct. (Theochem) 681, 21-25 (2004)

30. Khan, M.S., Khan, Z.H.: Ab initio and semiempirical study of structure and electronic spectra of hydroxy substituted naphthoquinones. Spectrochim. Acta. A. 61, 777-790 (2005)

31. Margulis, V.A., Muryumin, E.E., Tomilin, O.B.: - Atomic Hydrogen Adsorption on Boron Nitride Nanotube Surfaces. In: Veziroglu, T.N., Zaginalichenko, S.Y., Schur, D.V., Baranowski, B., Shpak, A.P., Skorokhod, V.V., Kale, A. (eds.) Hydrogen Materials Science and Chemistry of Carbon Nanomaterials, vol. 3, pp. 275-278. Springer, Dordrecht (2007)

32. Goel, R., Siddiqi, W.A., Ahmed, B., Khan, M.S., Chaubey, V.M.: Synthesis characterization and corrosion inhibition efficiency of N-C2 (2E)-2-[4(dimethylamino) benzylidene] hydrazinyl 2-oxo ethyl benzamide on mild steel. Desalination. 263, 45-57 (2010)

33. HyperChem(TM) Professional 7.52, Hypercube, Inc., 1115 NW 4th Street, Gainesville, Florida 32601, USA

34. Gorelsky, S.I.: AOMix: Program for Molecular Orbital Analysis. Ottawa, University of Ottawa (2007)

35. Gorelsky, S.I., Lever, A.B.P.: Electronic structure and spectra of ruthenium diimine complexes by density functional theory and INDO/S. Comparison of two methods. J. Organomet. Chem. 635, 187-196 (2001)
36. Margine, E.R., Crespi, V.H.: Universal behavior of nearly free electron states in carbon nanotubes. Phys. Rev. Lett. 96, 196803-196806 (2006)

37. Farmanzabeh, D., Ghazanfary, S.: First principle electric field response of single-walled boron nitride nanotube: a case study of zigzag $(4,0)$ model. Struct. Chem. 20, 709-717 (2009)

doi:10.1186/2228-5326-2-7

Cite this article as: Khan and Khan: Retracted: Computational study of hydrogen adsorption on potassium-decorated boron nitride nanotubes. International Nano Letters 2012 2:7.

\section{Submit your manuscript to a SpringerOpen ${ }^{\odot}$ journal and benefit from:}

- Convenient online submission

- Rigorous peer review

- Immediate publication on acceptance

Open access: articles freely available online

- High visibility within the field

- Retaining the copyright to your article

Submit your next manuscript at springeropen.com 